\title{
Proteochemometric modelling coupled to in silico target prediction: an integrated approach for the simultaneous prediction of polypharmacology and binding affinity/potency of small molecules
}

\author{
Shardul Paricharak ${ }^{1,2+}$, Isidro Cortés-Ciriano ${ }^{3 \dagger}$, Adriaan P IJzerman², Thérèse E Malliavin ${ }^{3 *}$
} and Andreas Bender ${ }^{1 *}$

\begin{abstract}
The rampant increase of public bioactivity databases has fostered the development of computational chemogenomics methodologies to evaluate potential ligand-target interactions (polypharmacology) both in a qualitative and quantitative way. Bayesian target prediction algorithms predict the probability of an interaction between a compound and a panel of targets, thus assessing compound polypharmacology qualitatively, whereas structure-activity relationship techniques are able to provide quantitative bioactivity predictions. We propose an integrated drug discovery pipeline combining in silico target prediction and proteochemometric modelling (PCM) for the respective prediction of compound polypharmacology and potency/affinity. The proposed pipeline was evaluated on the retrospective discovery of Plasmodium falciparum DHFR inhibitors. The qualitative in silico target prediction model comprised 553,084 ligand-target associations (a total of 262,174 compounds), covering 3,481 protein targets and used protein domain annotations to extrapolate predictions across species. The prediction of bioactivities for plasmodial DHFR led to a recall value of $79 \%$ and a precision of 100\%, where the latter high value arises from the structural similarity of plasmodial DHFR inhibitors and T. gondii DHFR inhibitors in the training set. Quantitative PCM models were then trained on a dataset comprising 20 eukaryotic, protozoan and bacterial DHFR sequences, and 1,505 distinct compounds (in total 3,099 data points). The most predictive PCM model exhibited $R_{0}^{2}$ test and RMSE $\mathrm{E}_{\text {test }}$ values of 0.79 and $0.59 \mathrm{plC} \mathrm{C}_{50}$ units respectively, which was shown to outperform models based exclusively on compound $\left(R_{0}^{2}\right.$ test $\left./ R_{M S E_{\text {test }}}=0.63 / 0.78\right)$ and target information $\left(R_{0}^{2}\right.$ test $\left./ \mathrm{RMSE}_{\text {test }}=0.09 / 1.22\right)$, as well as inductive transfer knowledge between targets, with respective $R_{0}^{2}$ test and RMSE $E_{\text {test }}$ values of 0.76 and $0.63 \mathrm{plC}_{50}$ units. Finally, both methods were integrated to predict the protein targets and the potency on plasmodial DHFR for the GSK TCAMS dataset, which comprises 13,533 compounds displaying strong anti-malarial activity. 534 of those compounds were identified as DHFR inhibitors by the target prediction algorithm, while the PCM algorithm identified 25 compounds, and 23 compounds (predicted $\mathrm{plC}_{50}>7$ ) were identified by both methods. Overall, this integrated approach simultaneously provides target and potency/affinity predictions for small molecules.
\end{abstract}

Keywords: Target prediction, chemogenomics, proteochemometrics, QSAR, DHFR, plasmodium falciparum

\footnotetext{
*Correspondence: therese.malliavin@pasteur.fr; ab454@cam.ac.uk

${ }^{\dagger}$ Equal contributors

${ }^{3}$ Unité de Bioinformatique Structurale, Institut Pasteur and CNRS UMR 3825,

Structural Biology and Chemistry Department, 25-28, rue du Dr. Roux, 75724

Paris, France

${ }^{1}$ Department of Chemistry, Centre for Molecular Science Informatics,

University of Cambridge, Lensfield Road, CB2 1EW Cambridge, UK

Full list of author information is available at the end of the article
}

\section{Chemistry Central}

(C) 2015 Paricharak et al.; licensee Springer. This is an Open Access article distributed under the terms of the Creative Commons Attribution License (http://creativecommons.org/licenses/by/4.0), which permits unrestricted use, distribution, and reproduction in any medium, provided the original work is properly credited. 


\section{Background}

In recent years it has been demonstrated that drugs exert their therapeutic effect by modulating more than one target, in fact six on average [1]. Therefore, the early evaluation of the bioactivity profiles of lead compounds is essential for the success in developing new drugs, although efficacy is sometimes attained by the inhibition of single targets, e.g. viral proteins. Similarly, understanding drug polypharmacology can help in anticipating drug adverse effects [2].

In parallel, the availability of public bioactivity databases has enabled the application of large-scale chemogenomics techniques to, among others, predict protein targets for small molecules, and to predict their affinity on therapeutically interesting targets [3]. These techniques capitalize on bioactivity data to infer relationships between the compounds, encoded with numerical descriptors, and their targets, which can be represented as labels in a classification model or explicitly encoded by e.g. protein or amino acid descriptors [4].

In silico target prediction algorithms assess potential compound polypharmacology through the computational evaluation of the (functionally unrelated) targets modulated by a given compound, or its selectivity to species-specific targets, as they predict the probability of interaction of that compound with a panel of targets [5]. Initially, target prediction models were developed using Laplacian-modified Naïve Bayesian classifiers [6] and the Winnow algorithm [7]. Later, Keiser et al. [8] developed a model which related biological targets based on ligand similarities and ranked the significance of the resulting similarity scores using the Similarity Ensemble Approach (SEA), followed by Wale and Karypis [9] who applied SVM and ranking perceptron algorithms to rank targets for a given compound. More recently, Koutsoukas et al. [10] compared the performance of both the Naïve Bayesian and Parzen-Rosenblatt Window classifiers, concluding that the overall performance of both methods is comparable though differences were found for certain target classes.

The ligand-target prediction methods described above generally predict the likelihood of interaction with a target, and they do not predict compound affinity or potency (e.g. $\mathrm{K}_{\mathrm{i}}$ or $\mathrm{IC}_{50}$ ). On the other hand, quantitative bioactivity prediction techniques, e.g. proteochemometric modelling (PCM) [3], predict the potency or affinity for compoundtarget pairs, normally in the form of $\mathrm{pIC}_{50}$ or $\mathrm{pK}_{\mathrm{i}}$ values. PCM combines information from compounds and related targets, e.g. orthologs, in a single machine learning model $[3,11]$, which enables the simultaneous modelling of chemical and biological information, and thus the prediction of compound affinity and selectivity across a panel of targets. Nonetheless, the effects of a compound at the cellular or the organism level are poorly understood in this case, as these methods cannot account for the interactions of a compound with other unrelated targets, which are not captured in the PCM model.

Given the limitations of both purely qualitative and purely quantitative bioactivity modelling approaches, in the current work, we propose an integrated drug discovery approach, combining in silico target prediction for the qualitative large-scale evaluation of compound bioactivity, and PCM for the quantitative prediction of compound potency. The proposed approach was evaluated on the discovery of DHFR inhibitors for Plasmodium falciparum ( $P$. falciparum), the causative agent of the most dangerous form of malaria [12]. Whilst there are multiple antimalarial drugs on the market, resistance to anti-malarial drugs is on the rise [13,14], and there are only $21 \mathrm{com}-$ pounds in clinical or pre-clinical trials [15].

In order to combat the lack of novel drugs for malaria, big pharmaceutical companies have generated a wealth of phenotypic data, namely the GlaxoSmithKline (GSK) TCAMS dataset, as well as the Novartis-GNF Malaria Box $[16,17]$. Both datasets contain phenotypic readouts, describing how effective the compounds present in the datasets are in inhibiting the growth of $P$. falciparum. Nonetheless, none of them contain annotations about the P. falciparum target(s) involved, making it a challenge to elucidate the mode of action (MoA) of the compounds in the dataset, and hence, making the dataset difficult to interpret. This renders these datasets a very suitable case study for the algorithms we are presenting in this work.

In the context of malaria drug discovery, previous studies have applied machine learning algorithms to predict whether plasmodial proteins are secretory proteins based on their residue composition [18], and to predict the bioactivities of compounds against particular plasmodial targets $[19,20]$. These approaches, though, did not account for the polypharmacology of anti-malarial compounds.

To overcome the limitations of these methods, we now integrate both in silico target prediction and PCM in a unified drug discovery approach. As illustrated in Figure 1, the target prediction algorithm used in this study, trained on approximately 553,084 bioactivity data points spanning 3,481 targets, used a domainbased similarity metric between targets to extrapolate target predictions from one species to another. Nonplasmodial targets were then extrapolated to plasmodial targets. Besides, the PCM model was trained on a dataset composed of 20 eukaryotic, protozoan and bacterial DHFR sequences, and of 1,505 different DHFR inhibitors and a total of 3,099 data points. To exploit the complementarity of the two prediction methods, in silico target prediction was used to predict MoA hypotheses for the anti-malarial compounds in the GSK TCAMS phenotypic dataset, 


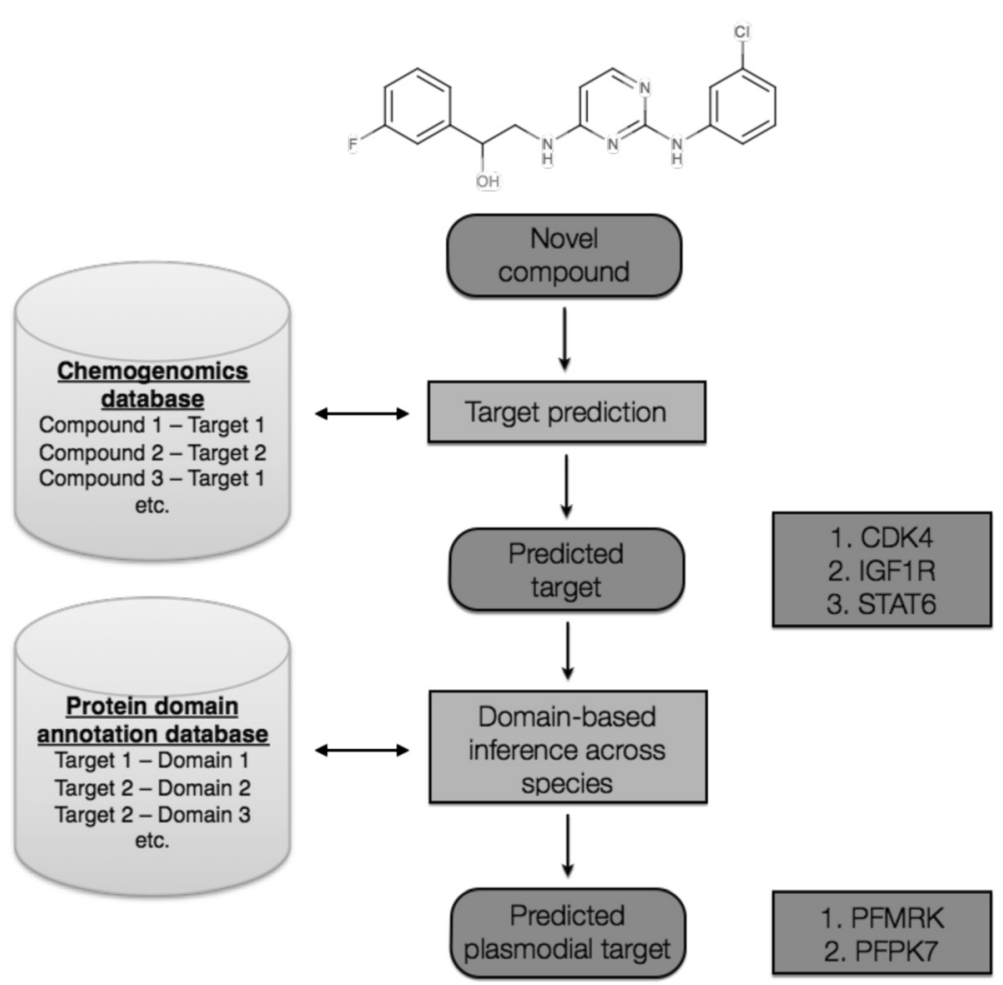

Figure 1 Schematic overview of in silico target prediction and domain-based extrapolation workflow. The conventional in silico target prediction approach [10] is extended in this study by using protein domain annotations to extrapolate from non-plasmodial target predictions to protein target predictions in P. falciparum. This concept is generally applicable across organisms, in particular to those for which little bioactivity data is currently available.

whereas PCM was employed to quantify compound potency $\left(\mathrm{pIC}_{50}\right)$.

\section{Methods}

Exploratory principal component analysis (PCA) of PCM and target prediction datasets

A PCA was performed for compounds contained in the PCM dataset, as well as for those annotated on P. falciparum and $T$. gondii in the target prediction dataset. The Spearman's rank correlation coefficient was calculated for all pairs of compound descriptors, based on both physicochemical descriptors and Morgan fingerprints, thus defining a square correlation matrix. The PCA analysis was performed on this matrix in order to avoid the direct application of PCA on binary descriptors, i.e. Morgan fingerprints. Visualization was performed using $\mathrm{R}$ and Vortex [21].

\section{Target prediction}

\section{Training dataset}

Bioactivity data were extracted from ChEMBL16 [22] according to the protocol described by Koutsoukas et al. [10]. The extracted data contained approximately 4 million bioactivities covering approximately 8,000 biomolecular targets, of which approximately 4,000 targets were proteins
$[22,23]$. Compound-target pairs were selected according to the following criteria: (i) $\mathrm{K}_{\mathrm{i}}, \mathrm{K}_{\mathrm{d}}, \mathrm{IC}_{50}$ or $\mathrm{EC}_{50}$ bioactivity values equal to or lower than $10 \mu \mathrm{M}$, and (ii) targets annotated with a confidence score of 8 (homologous single protein target assigned) or 9 (direct single protein target assigned). Subsequently, ligand structures were processed with the ChemAxon standardizer version 5.12.0 [24], with the following options: "Remove fragment", "Neutralize", "Aromatize", "Clean2D", "Tautomerize" and "Remove explicit hydrogens". After standardization, the entries with ligands annotated against multiple targets were detected based on their canonical SMILES and removed using custom Perl scripts, resulting in a training set of 553,084 instances $(262,174$ compounds) covering 3,481 protein targets (Additional file 1: Supplementary Information SI 1). The bioactivity data of $P$. falciparum (1,513 instances 1,379 compounds covering 41 protein targets) was omitted from this dataset for training purposes. InterPro [25] domain annotations were retrieved for all protein targets using the Uniprot database [26].

\section{P. falciparum dataset}

The $P$. falciparum dataset was built using the same criteria as described above, resulting in a set comprising 41 $P$. falciparum targets and 1,379 compounds. In addition, 
all annotated and reviewed $P$. falciparum targets from Uniprot were downloaded, resulting in a total of $148 P$. falciparum protein targets. Finally, InterPro domain annotations were retrieved for all protein targets using the Uniprot database (Additional file 2: Supplementary Information SI 2).

\section{GSK TCAMS dataset}

Approximately 2 million compounds present in GSK's screening collection have been tested in vitro by GSK for inhibitors of $P$. falciparum's intraerythrocytic cycle based on growth inhibition assays [17]. Briefly, assays were performed on both the reference laboratory strain 3D7, as well as on the multidrug resistant strain Dd2, where parasite growth was evaluated using LDH activity [17]. 19,451 compounds were identified as primary hits inhibiting the 3D7 strain growth by more than $80 \%$ at $2 \mu \mathrm{M}$ concentration, of which 13,533 compounds displayed $80 \%$ or higher inhibition of parasite growth in at least 2 of the 3 assay runs in independent follow-up experiments. Hence, these 13,533 compounds were considered as confirmed inhibitors (confirmation rate $>70 \%$ ) and used in the present study.

\section{Descriptors}

A circular fingerprint implementation, Molprint2D [27,28] was used for encoding molecular structures, since this method has previously been shown to capture structural aspects related to bioactivity better than most other descriptors in comparative studies [29]. This descriptor is based on count vectors of heavy atoms present at a topological distance from each heavy atom of a molecule [28]. For the present study, the pybel implementation was used [30].

\section{Target prediction algorithm}

A multiclass Laplacian-modified Naïve Bayesian classifier, as described by Nigsch et al. [7] and later implemented by Koutsoukas et al. [10] was implemented to classify the bioactivity dataset and to be able to predict targets for novel compounds. For the query molecule $\boldsymbol{x}$, consisting of a set of $n$ Molprint2D features $f_{i}$, the likelihood to be active against a protein target $\omega_{\alpha}$ was calculated using the following equation:

$$
S_{\boldsymbol{\omega}_{\alpha}}(\boldsymbol{x})=\sum_{i=1}^{n} \log \left(\frac{N_{i, \omega_{\alpha}}+1}{N_{i} \times p\left(\omega_{\alpha}\right)+1}\right)+\log \left(\frac{\prod_{i=1}^{d} p\left(f_{i}\right)}{p(\boldsymbol{x})}\right)
$$

where $S_{\omega_{\alpha}}(\boldsymbol{x})$ is the logarithmic likelihood score (proportional to the likelihood of bioactivity), $N_{i, \omega_{\alpha}}$ is the total number of occurrences of feature $f_{i}$ in protein class $\omega_{\alpha}$ and $N_{i}$ is the total number of occurrences of feature $f_{i}$ in the entire training set. Furthermore, $p\left(\omega_{\alpha}\right)$ is the prior probability of protein class $\omega_{\alpha}$. The prior probability quantifies how likely a compound is active against protein target $\omega_{\alpha}$ in the absence of any feature information. It can be calculated as follows:

$$
p\left(\omega_{\alpha}\right)=\frac{N_{\omega_{\alpha}}}{N}
$$

where $N_{\omega_{\alpha}}$ is the number of instances (i.e. bioactivities) in class $\omega_{\alpha}$ and $N$ is the total number of instances. The predictive performance of this model was assessed in terms of average class-specific recall and precision. Only target classes with 20 or more data points in the $P$. falciparum dataset were considered as suitable for testing due to a sufficient number of data points, resulting in a total of 16 target classes.

\section{Domain-based extrapolation to P. falciparum targets}

For each analyzed compound, the top $n$ ranked predicted targets were compared to all 148 P. falciparum targets in terms of their InterPro domain composition. P. falciparum targets with an InterPro domain Tanimoto similarity above a variable cut-off were considered as predicted, but were not ranked. The cut-off value varied between 0.5 and 1 , where 1 means that only orthologous proteins are considered. The target prediction and domain-based extrapolation pipeline are illustrated in Figure 1. The domain extrapolation extends the target prediction approach $[10,31]$ by using InterPro protein domain annotations to extrapolate from predicted nonplasmodial targets to $P$. falciparum targets. This is conceptually similar to a previously reported study for extrapolating bioactivities between species [32], and its application to M. tuberculosis [33].

The inclusion of plasmodial DHFR (CHEMBL1939) bioactivity data was expected to drastically improve the performance, and this was tested in the following way. A 2-fold cross validation (CV) was performed: the instances annotated on plasmodial DHFR were split into 2 half subsets, where one subset was added to the training set and the other half was used as a test set (and vice versa).

\section{Proteochemometric modelling}

Dataset

$\mathrm{IC}_{50}$ values with a confidence score of 8 or 9 for 20 DHFR sequences (Table S3) were retrieved from ChEMBL16 [22] and this initial dataset comprised 5,827 data points. In the cases where a compound-target combination had more than one annotated bioactivity value, the set of bioactivities was replaced by its mean value. This procedure is robust, because the standard deviation of the differences was smaller than $0.1 \mathrm{pIC}_{50}$ unit in more than $90 \%$ of the cases (Additional file 3: Figure S1). This resulted in a dataset including 3,099 distinct compound-target combinations. The matrix completeness of the dataset, calculated as the 
number of compound-target combinations present in the dataset over the total number of possible compoundtarget combinations, was $10.3 \%$. Compounds included in the PCM dataset were not present in the target prediction dataset.

\section{Descriptors}

Chemical structures were standardized and cleaned with the function StandardiseMolecules of the R package camb using the default parameters [34] and PaDEL descriptors (1-D and 2-D). Morgan fingerprints were calculated in the same environment. The function $A A_{-}$Descs was used to calculate amino acid descriptors (3 Z-scales). To describe the target space, the residues in the binding site of human DHFR (PDB ID: 1OHJ [35]) within a sphere of $10 \AA$ centered around the ligand were selected. The corresponding residues for the other 19 proteins were obtained from a sequence alignment realized with Clustal Omega [36]. The dataset is available in Additional file 4 (Supplementary Information SI 3).

\section{Proteochemometric modelling}

All descriptor values were centered to zero mean and scaled to unit variance. The dataset was split into six subsets, five of which were used to train models, and the sixth, test set, was withheld to assess the predictive ability of the models [37]. The hyperparameter values for all PCM models were optimized by 5 -fold cross validation [38]. To assess both model predictive ability and performance, the $\mathrm{pIC}_{50}$ values for the test set were predicted, thus providing the external validation by calculating $\mathrm{RMSE}_{\text {test }}$ and $R^{2}{ }_{0}$ test between the observed and the predicted $\mathrm{pIC}_{50}$ values:

$$
\begin{aligned}
& R_{0 \text { test }}^{2}=1-\frac{\sum_{i=1}^{N}\left(y_{i}-\hat{y}_{i}^{r 0}\right)^{2}}{\sum_{i=1}^{N}\left(y_{i}-\bar{y}\right)^{2}} \\
& R M S E=\sqrt{\frac{(y-\hat{y})^{2}}{N}}
\end{aligned}
$$

where $N$ represents the size of the test set, $y_{i}$ the observed, $\hat{y}_{i}$, the predicted, and $\bar{y}$ the average $\mathrm{pIC}_{50}$ values of those datapoints included in the test set, and $\hat{y}_{i}^{r o}=s \hat{y}$, with $s=\sum y_{i} \hat{y}_{i} / \sum \hat{y}_{i}^{2}$. Both internal (RMSE $\mathrm{int}_{\text {int }}$ and $R_{\text {int }}^{2}$ ) and external validation $\left(\mathrm{RMSE}_{\text {test }}\right.$ and $\left.R^{2}{ }_{\mathrm{o} \text { ext }}\right)$ were assessed according to the criteria proposed by Tropsha et al. $[39,40]$ and calculated using the Validation function of the R package camb [34].

In order to assess whether the combination of compound and target information in a single PCM model constitutes an advantage with respect to one-space (ligand space and target space) models, two validation scenarios were explored. Firstly, a Family QSAR model [41] was trained exclusively on compound descriptors. High performance of this model is expected in cases where the bioactivities of the same compound on different targets are highly correlated. Secondly, the Family QSAM [41] model was trained on target descriptors only. In this case, high performance would indicate that the activities of a diverse set of compounds are correlated on a panel of targets. Thus, compound activities would largely depend on the target, and to a much lesser extent on the ligand structures.

Additionally, an inductive transfer PCM model (PCM IT) was trained to assess whether the performance of PCM models arises from explicit learning (EL), where the knowledge is extracted from target descriptors, or inductive transfer (IT). In IT the knowledge acquired when predicting compound bioactivities on a given target is exploited to predict the bioactivity of those compounds on another target [41]. In the PCM IT model, targets were described with identity fingerprints (IFP), which are calculated as follows:

$$
\operatorname{IFP}(i, j)=\delta(i-j)\left(i, j \in 1, \ldots, N_{\text {targets }}\right)
$$

where $\delta$ is the Kronecker delta function and $\mathrm{N}_{\text {targets }}$ the number of distinct targets. The performance of the models was assessed on a per target basis by training ten PCM models, each on a different subset of the whole dataset. Subsequently, $\mathrm{RMSE}_{\text {test }}$ and $R^{2}{ }_{O}$ test values were calculated on subsets of the test set grouped by target.

\section{Machine learning implementation}

Support Vector Machines (SVM) [42], Gradient Boosting Machines (GBM) [43], Gaussian Processes (GP) [44], and Random Forest (RF) [45] models were built with the $\mathrm{R}$ package camb $[34,46]$. The target prediction algorithm was implemented in Perl.

\section{Results and discussion \\ Exploratory analysis of PCM and target prediction datasets}

A PCA (Figure 2) was performed for the compounds annotated to be active against plasmodial DHFR and those active against $T$. gondii DHFR. The first two principal components explain $72.73 \%$ of the variance. In the two dimensions visualized for the descriptor space used here, the plasmodial inhibitors cover a substantial portion of the chemical space occupied by the $T$. gondii DHFR inhibitors. However, there are still a number of clusters of T. gondii DHFR inhibitors that occupy novel space not covered by plasmodial inhibitors. Compounds from these clusters contain bicyclic ring systems (shown in red boxes in Figure 2). On the other hand, there are also clusters of plasmodial inhibitors that occupy space not covered by $T$. gondii inhibitors: these plasmodial inhibitors do not contain bicyclic rings, but instead contain unfused rings (5 scaffolds identified shown in green 


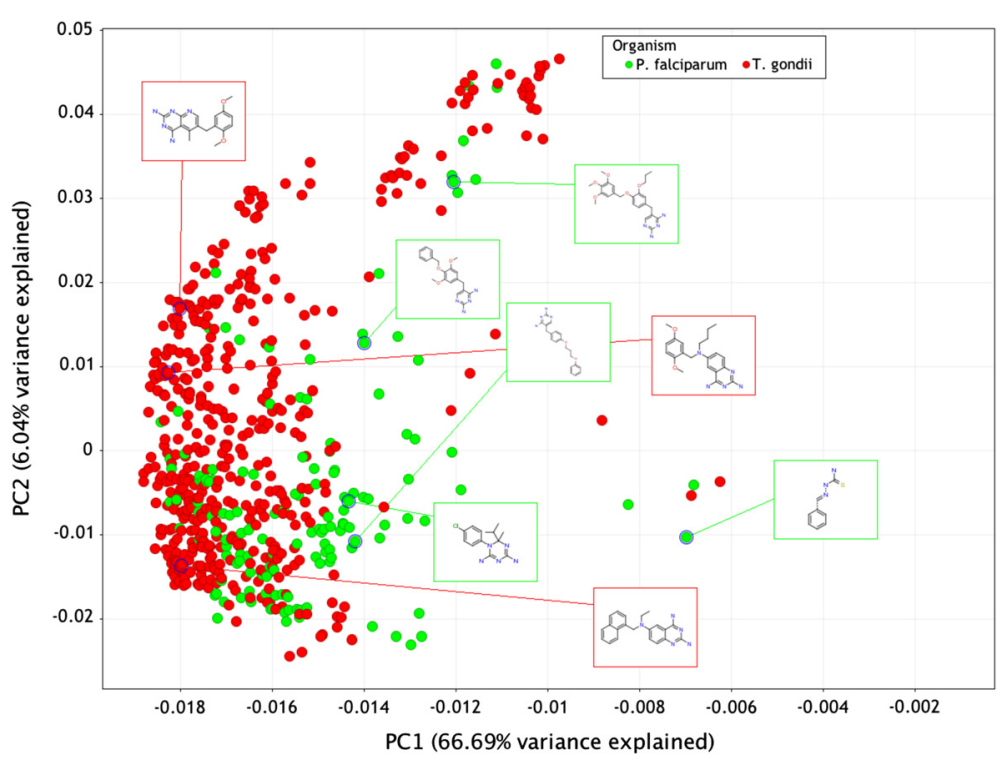

Figure 2 PCA of the compounds annotated as actives against plasmodial DHFR (green) as well as T. gondii DHFR (red). Overall, plasmodial DHFR inhibitors cover a substantial portion of the chemical space occupied by T. gondii DHFR inhibitors. However, some clusters of T. gondii DHFR inhibitors are located in additional chemical space not covered by the plasmodial inhibitors (red boxes). These clusters contain compounds with bicyclic ring systems. By contrast, plasmodial inhibitors only contain unfused rings (green boxes). These observations explain why recall is low $(\sim 35 \%)$ when plasmodial DHFR inhibitors are excluded from the training set: T. gondii inhibitors do not cover all relevant chemical space, particularly the space occupied by compounds with unfused ring systems.

boxes in Figure 2). In addition to the previous analysis, a PCA was also performed for the compounds present in the PCM dataset (Additional file 3: Figure S2), where the first two principal components explained $51.77 \%$ of the variance. Clusters contain compounds whose bioactivities on several targets are included in the dataset, thus indicating that compounds are overall structurally similar across the 20 DHFR sequences considered.

\section{Application of target prediction for MoA prediction}

The performance of the target prediction algorithm was assessed for varying values of $n$, which represents the top number of non-plasmodial predictions considered for extrapolation (Additional file 3: Figure S3). It can be seen that performance varies widely across target classes: for most targets, including all aminopeptidases, calciumdependent protein kinase 1, protein kinase Pfmrk, glucose-6-phosphate-1-dehydrogenase, dihydroorotate dehydrogenase, dUTP pyrophosphatase and enoyl-acylcarrier protein reductase, performance is low, with both recall and precision values below $30 \%$. For a small number of targets, however, the performance is much higher, with recall values up to $\sim 60 \%$ and precision values up to $100 \%$. Further investigation revealed that the targets for which the prediction algorithm performed well (plasmepsin 1 and 2, histone deacetylase, DHFR and to a lesser extent, falcipain 2 ) were plasmodial orthologs of non-plasmodial protein targets. This finding is in agreement with previous studies, which have used orthologous proteins to extrapolate the prediction of bioactivities between target classes across species such as P. falciparum and M. tuberculosis [47,48]. However, these previous studies have not combined target prediction with PCM for MoA analysis, which is precisely the novelty of the approach presented here.

\section{Target prediction performance for plasmodial DHFR}

The predictive performance of the target prediction algorithm was further investigated for the plasmodial target DHFR, where all 145 instances annotated on plasmodial DHFR were used as a test set. The top $n$ predicted non-plasmodial targets were considered ( $n$ varied in the 112 range), after which these targets were extrapolated to plasmodial targets (section "Domain-based extrapolation to P. falciparum targets" in Materials and Methods). For $n$ in the 1-3 range, the recall values are $0 \%, 2.8 \%$ and $14,5 \%$, respectively, whereas for $n$ in the 4-7 range, the recall values are around $35 \%$. The 2 -fold CV resulted in a recall value of $79 \%$. These results indicate that despite the fact that the training set did not contain any plasmodial bioactivity data, the model is still able to predict compounds active against plasmodial DHFR with 100\% precision, based on bioactivity data for orthologous proteins across other species. The high precision value arises from the structural similarity of plasmodial DHFR inhibitors and T. gondii DHFR inhibitors in the training set (the average MOLPRINT2D pairwise similarity between the $T$. gondii inhibitors and the plasmodial inhibitors was $16 \%$, whereas the average pairwise similarity within the plasmodial dataset and the T. gondii dataset was 
$19 \%$ and $18 \%$ respectively). These results show the added benefit of incorporating domain-based extrapolation for target prediction purposes.

In addition, we found that varying the domain Tanimoto similarity cut-off between 0.5 and 1 did not alter the performance. Hence, in order to maintain high precision, a stringent domain Tanimoto similarity cut-off of 1 (i.e. requiring a $100 \%$ overlap in domain presence and absence between two proteins) was chosen and the top $n$ predicted non-plasmodial targets considered was set to 4 for further analysis. Further investigation of the extrapolation from non-plasmodial targets to plasmodial targets revealed that only one protein class ( $T$. gondii DHFR) was responsible for the extrapolation of predicted activities to plasmodial DHFR. As described earlier, there are clusters of T. gondii DHFR inhibitors that do not contain any plasmodial DHFR inhibitors (scaffolds identified in these clusters are shown in red boxes - Figure 2 and clusters of plasmodial inhibitors that occupy space not covered by $T$. gondii inhibitors ( 5 scaffolds identified shown in green boxes in Figure 2). Hence, for these clusters there is no overlap in scaffolds between both datasets. These observations explain the low recall of the model at this stage: plasmodial DHFR inhibitors located outside the space covered by $T$. gondii DHFR inhibitors are not retrieved by the model, thereby increasing the number of false negatives, whereas the plasmodial DHFR inhibitors that are present in the chemical space shared by inhibitors from both species are predicted with very high precision.

Adding plasmodial DHFR data to the training set drastically increased performance, more than doubling recall values to $79 \%$, whereas precision values remained 100\% (Figure 3 - 2-fold CV). Hence, this observation arises from the fact that the chemical space of the plasmodial DHFR inhibitors adds additional information corresponding to 5 new scaffolds (as highlighted in green boxes in Figure 2) to the model. However, despite the very high precision value achieved $(100 \%)$, there is a drawback: given the great increase in recall value when novel scaffolds are added to the dataset, the model is only able to correctly predict bioactivities for compounds with scaffolds that are already present in the training data. Hence, a diverse set of molecules is required in the training set in order to optimize recall values of the model. Given the benefit of both domainbased extrapolation and using plasmodial DHFR bioactivity data for model training, all plasmodial DHFR data were included in the training set for further MoA

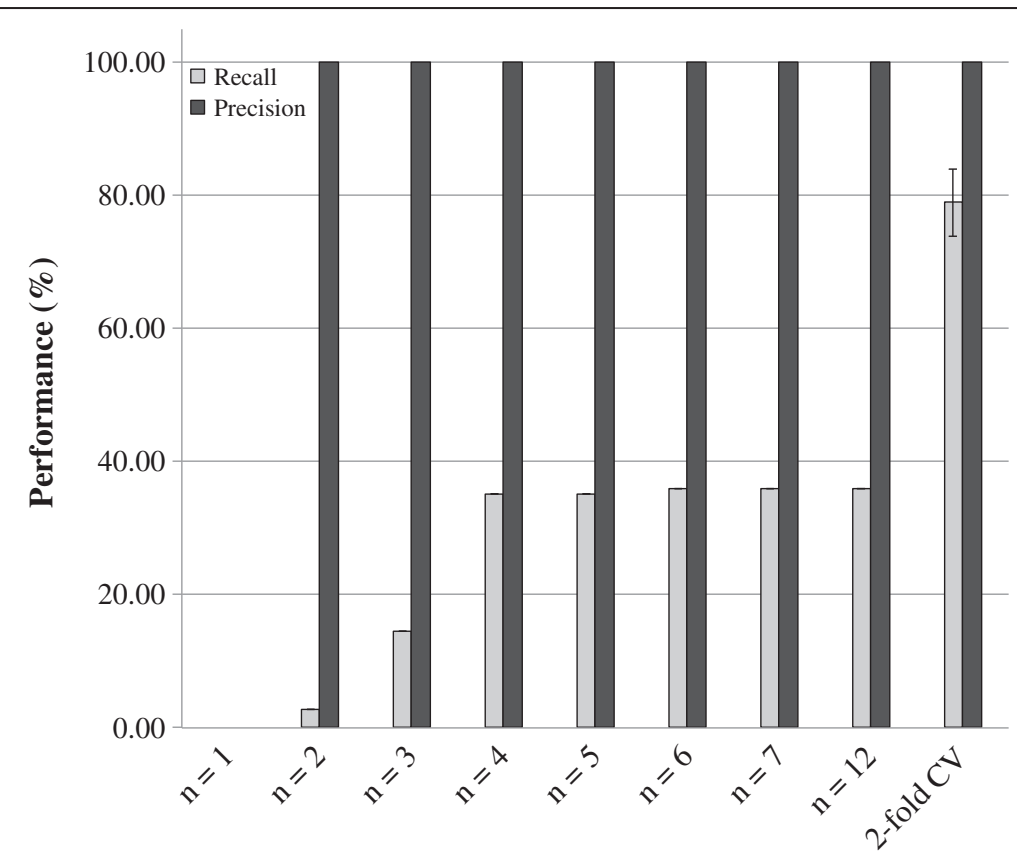

Figure 3 Performance of the DHFR target prediction model compared across a number of parameters. 145 data points annotated against plasmodial DHFR were used as a test set to assess the performance of the target prediction model. The top $n$ predicted non-plasmodial targets were considered ( $n$ was varied for values between 1 and 12), after which these targets were extrapolated to plasmodial targets. When $n$ increases, recall values rise up to $36 \%$ (with recall values of $\sim 35 \%$ for $n=3$ and $n=4$ ). On the other hand, precision values are $100 \%$ for $n \geq 2$. The high precision values are likely to be explained by the fact that plasmodial DHFR inhibitors and T. gondii DHFR inhibitors occupy the same chemical space. In addition to varying the parameter $n$, we performed a 2-fold cross validation (averaged over 20 randomizations), which resulted in a drastic improvement as a recall value of $79 \%$ was achieved (with a standard deviation of $10.1 \%$, which is shown as an error bar). These results show that domain-based extrapolations have added value to the prediction algorithm (correct predictions are made even when bioactivity data on plasmodial DHFR is not present in the training set) and that including plasmodial DHFR bioactivity data in the training set can drastically improve recall values. 
Table 1 PCM, Family QSAR and Family QSAM performance on the PCM dataset

\begin{tabular}{lllll}
\hline & $\mathbf{R}_{\mathbf{C V}}$ & $\mathbf{R M S E}_{\mathbf{C V}}$ & $\mathbf{R}_{\mathbf{0}}^{\mathbf{2}} \mathbf{\text { t }}$ & $\mathbf{R M S E}_{\text {ext }}$ \\
\hline GBM PCM & 0.75 & 0.64 & 0.79 & 0.59 \\
GP PCM & 0.75 & 0.65 & 0.76 & 0.63 \\
RF PCM & 0.74 & 0.66 & 0.77 & 0.62 \\
SVM PCM & 0.76 & 0.63 & 0.77 & 0.62 \\
Family QSAM & 0.07 & 1.24 & 0.09 & 1.22 \\
Family QSAR & 0.61 & 0.80 & 0.63 & 0.78 \\
Inductive Transfer & 0.72 & 0.68 & 0.76 & 0.63
\end{tabular}

Abbreviations: QSAM Quantitative Structure-Activity Modelling, QSAR Quantitative Structure-Activity Relationship, GBM Gradient Boosting Machine, GP Gaussian Process, RF Random Forest, SVM Support Vector Machine.

$P C M$, with $R_{0}^{2}$ test and RMSE both Family QSAR, with $\mathrm{R}_{0}^{2}$ test and RMSE $\mathrm{test}_{\text {t }}$ values of 0.63 and $0.78 \mathrm{plC}_{50}$ units, respectively, and Family QSAM, with with $\mathrm{R}_{0}^{2}$ test and $\mathrm{RMSE}_{\text {test }}$ values of 0.09 and $1.22 \mathrm{plC}_{50}$ units, respectively.

prediction of the GSK TCAMS phenotypic dataset in order to optimize recall values.

\section{PCM model validation}

The four algorithms used in this study (GBM, GP, RF and SVM) displayed similar performance on this dataset as the ranges of RMSE $E_{\text {test }}$ and $R_{0}^{2}$ test differences are 0.04 $\mathrm{pIC}_{50}$ and 0.02 units, respectively. The GBM model exhibited the highest predictive ability with $\mathrm{R}_{0}^{2}$ test and $\mathrm{RMSE}_{\text {test }}$ values of 0.79 and $0.59 \mathrm{pIC}_{50}$ units respectively. Both internal and external validation metrics are given in Table 1.

To ensure that the model's predictive ability was not the consequence of spurious correlations in the data, we trained ten GBM models with an increasingly higher percentage of the $\mathrm{pIC}_{50}$ values randomized. Additional file 3: Figure S4 shows the performance of the ten models, quantified by the RMSE $E_{\text {test }}$ and $R_{0}^{2}$ test values as a function of the level of randomization of the bioactivity values. The intercept was zero or negative when $\sim 40 \%$ of the response variable was randomized (Additional file 3: Figure S4A).
Therefore, the relationship established by the PCM models between the descriptor space and the bioactivity values is not a consequence of chance correlations [49].

\section{PCM outperforms one-space models and IT on this dataset}

The Family QSAM model, trained on target descriptors only, displayed poor predictive ability with $\mathrm{RMSE}_{\text {test }}$ and $\mathrm{R}_{0}^{2}$ test values of $1.22 \mathrm{pIC}_{50}$ units and 0.09 , respectively (Table 1). By contrast, the Family QSAR model, trained on compound descriptors only, displayed satisfactory values for the statistical metrics according to our validation criteria, as the model exhibited $\mathrm{RMSE}_{\text {test }}$ and $\mathrm{R}_{0}^{2}$ test values of $0.78 \mathrm{pIC}_{50}$ units and 0.63 , respectively (Table 1 ). Hence, compound descriptors explain a large proportion of the variance, which may stem from the high correlation of the bioactivities of identical compounds against orthologs. Indeed, Additional file 3: Figure S5 depicts the correlation (RMSE: $0.95 \mathrm{pIC}_{50}$ units; $\mathrm{R}_{0}^{2}$ : 0.46 ) between the $\mathrm{pIC}_{50}$ values of the same compounds on different orthologs.

Furthermore, better performance is obtained for the GBM PCM model trained on amino acid descriptors and compound fingerprints, than for the GBM model trained on target identity fingerprints and compound fingeprints, with $\mathrm{RMSE}_{\text {test }}$ values of 0.59 vs. $0.63 \mathrm{pIC}_{50}$ units, respectively. This indicates that our selection of amino acid descriptors captured the binding site information of the different orthologs and thus allows explicit learning on this dataset (Table 1). Overall, these data suggest that the explicit inclusion of target information improves bioactivity prediction.

\section{Several high-affinity DHFR Inhibitors are identified by both target prediction and PCM}

The targets for which the target prediction model had a class-specific F-measure higher than $40 \%$ were selected, leading to a shortlist of 5 proteins, namely: plasmepsin

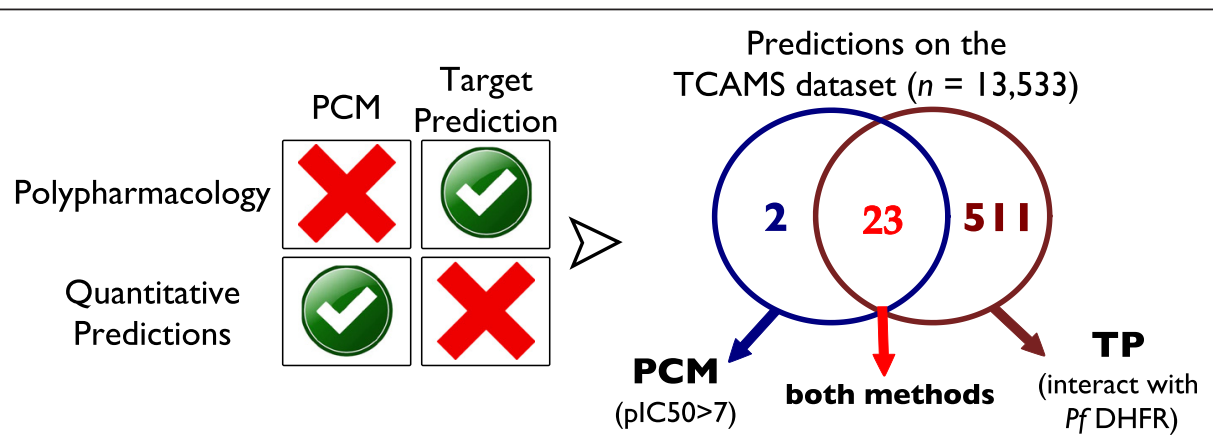

Figure 4 Complementarity between in silico target prediction and PCM. The target prediction algorithm predicted 534 compounds of the GSK TCAMS dataset to interact with DHFR, representing 3.95\% of the total number of compounds in this dataset. Out of these 534 compounds, the PCM model predicted 23 compounds to have a plC 50 value of 7 or greater. Therefore, the combination of both methods permits the assessment of compound polypharmacology and provides quantitative bioactivity predictions. 
1 and 2, histone deacetylase, DHFR and falcipain 2 (Additional file 3: Figure S6). Overall, a total of 1,291 plasmodial predictions were made for 1,017 compounds. DHFR is the most commonly predicted target, which represents 534 (41\%) of the total predictions, followed by plasmepsin 1 (280 predictions $-22 \%$ ) and plasmepsin 2 (273 predictions - 21 histone deacetylase (184 predictions - 14\%) and falcipain 2 (20 predictions $2 \%)$. Plasmodial DHFR has previously been proposed as a candidate target against resistant plasmodial strains [50]. In addition, the plasmepsin (1 and 2) and falcipain targets have previously been proposed as potential targets for anti- malarial therapy [51], due to their involvement in the hemoglobin catabolism that occurs during the erythrocytic stage of the malarial parasite life cycle (plasmepsin proteins and falcipain proteins), and to their involvement in erythrocyte invasion and erythrocyte rupture (falcipain proteins) [52]. Finally, plasmodial histone deacetylase has been proposed as a promising target for anti-malarial therapy due to its key role in regulating gene transcription, and it has been shown that histone deacetylase inhibitors are potent inhibitors of the growth of P. falciparum [53]. Hence, there is sufficient evidence for all 5 predicted proteins for being a potential target.

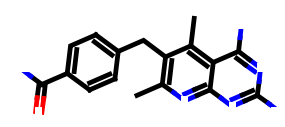

$137641 \uparrow$

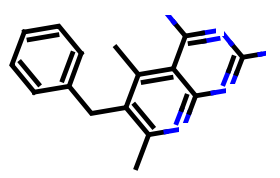

$137670 \uparrow$

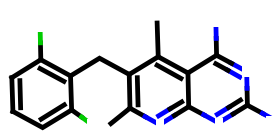

$137638 \uparrow$

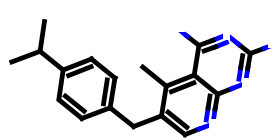

$138044 \uparrow$

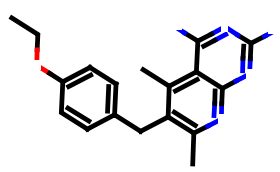

$137655 \uparrow$

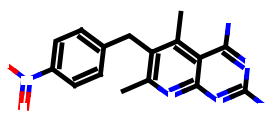

$137636 \uparrow$

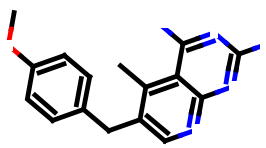

$137704 \uparrow$

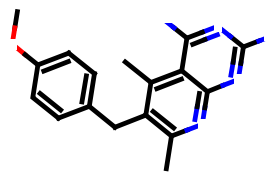

$137660 \uparrow$

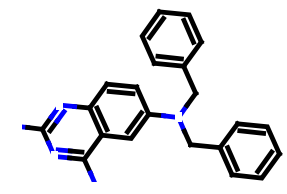

$137850 \uparrow$

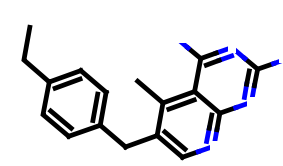

$137686 \uparrow$

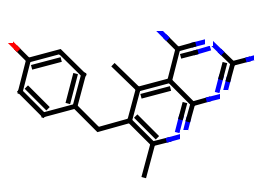

$137637 \uparrow$

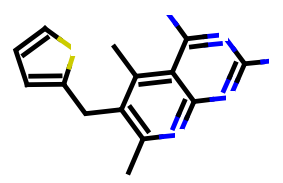

$137642 \uparrow$

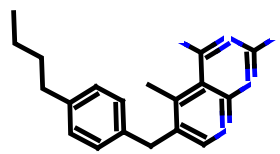

$138045 \uparrow$

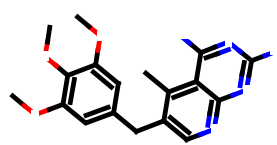

$138058 \uparrow$

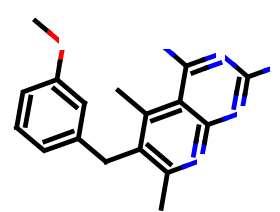

$137650 \uparrow$

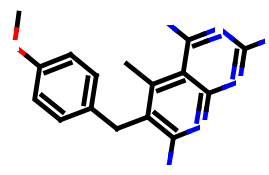

$138061 \uparrow$

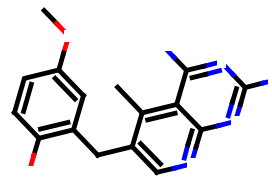

$137998 \uparrow$

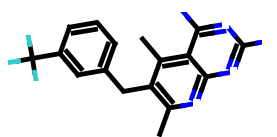

$137658 \uparrow$

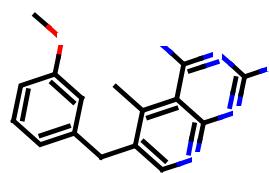

$138060 \uparrow$

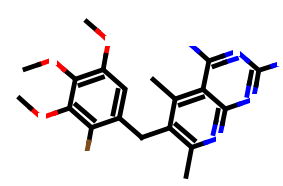

$137644 \uparrow$

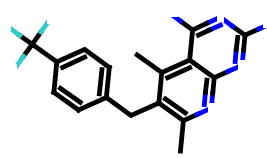

$137656 \uparrow$

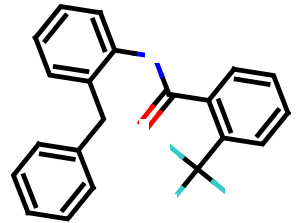

$123550 \downarrow$

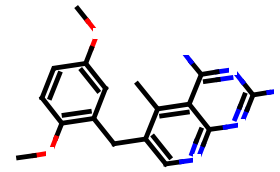

$137235 \uparrow$

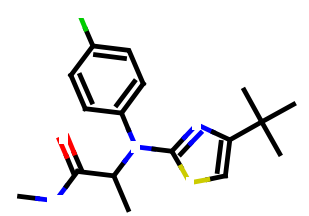

$125380 \downarrow$

Figure $\mathbf{5}$ Compounds predicted to interact with DHFR by the target prediction algorithm, and predicted by the PCM model to have a plC 50 value higher than $7 \mathrm{plC}_{50}$ units. Compound IDs correspond to the TCMDC identifier given in the original dataset. The 23 compounds for which the IDs are accompanied by an upward-pointing arrow were identified by the two methods. The two compounds predicted to have a pIC 50 value higher than 7 by the PCM model, but not predicted to interact with DHFR by the target prediction algorithm, are accompanied by a downward-pointing arrow. The 23 compounds predicted to be high-affinity DHFR inhibitors (upward-pointing arrows) share a common scaffold: a 5-methylpyrido [2,3-d]pyrimidine-2,4-diamine ring with an aryl substituent in the 6-position. Overall, it can be seen that these data indicate a high agreement between the target prediction algorithm and the PCM model to identify high-affinity DHFR inhibitors. 
In total, 534 compounds of the GSK TCAMS dataset were predicted to interact with DHFR, representing $3.95 \%$ of the total number of compounds in this dataset. Out of these 534 compounds, the predicted $\mathrm{pIC}_{50}$ values using PCM was 7 or greater for 25 compounds, between 6 and 7 for 92 compounds, and between 5 and 6 for 420 . None of the 534 compounds was predicted to be inactive on DHFR (Figure 4). Given that many of the compounds in ChEMBL are active in the low micromolar range, it is thus not surprising to obtain most of the predictions in this range [54].

Interestingly, 23 of the 25 compounds with a predicted $\mathrm{pIC}_{50}$ value higher than 7 were already predicted to interact with DHFR by the target prediction algorithm (Figure 4) at the exclusion of any other target. The analysis of chemical scaffolds in the 25 compounds shows that only 2 scaffolds were identified, as 22 out of the 25 compounds (Figure 5 - excluding compounds 137850, 123550 and 125380), share a common scaffold, namely: a 5-methylpyrido[2,3-d]pyrimidine-2,4-diamine ring with an aryl substituent in the 6-position. A methyl group or an amine group in the 7-position are also present in some compounds, such as 137637 and 138061, respectively. In all compounds with the common scaffold the aryl substituent is a phenyl ring with different substituents in the 3,4,5-positions, e.g. methoxy, hydroxy and carboxamide, except for compound 137642, which has 2-methyl-thiophene as aryl substituent.

Two additional compounds, 123550 and 125380 (Figure 5), predicted by PCM to display $\mathrm{pIC}_{50}$ values of 7.11 and 7.07, respectively, represent new scaffolds. Remarkably, these two scaffolds were neither present in the PCM nor in the target prediction training set. Taken together, our results indicate a high agreement between the target prediction algorithm and the PCM model to identify high-affinity DHFR inhibitors. Using both methods simultaneously, it is possible to give higher priority to the compounds that are identified by both methods.

\section{Conclusions}

In this study, the complementarity of in silico target predictions and proteochemometric modelling (PCM) was evaluated for the retrospective identification of $P$. falciparum DHFR inhibitors. The target prediction algorithm exhibited respective recall and precision values of $79 \%$ and $100 \%$ for plasmodial DHFR. The high precision value is explained by the structural similarity of plasmodial and the $T$. gondii DHFR inhibitors, which were part of the training set and were found to be relevant for extrapolation (the average MOLPRINT2D pairwise similarity between the $T$. gondii inhibitors and the plasmodial inhibitors was $16 \%$, whereas the average pairwise similarity within the plasmodial dataset and the T. gondii dataset was $19 \%$ and $18 \%$ respectively).
We showed that high-affinity inhibitors from the GSK TCAMS phenotypic dataset are independently identified by both methods: 534 compounds from the GSK TCAMS dataset were identified as DHFR inhibitors by the target prediction algorithm, whereas the PCM algorithm identified 25 high affinity compounds, 23 of which were already identified by the target prediction algorithm. The combination of both methods permits the assessment of compound polypharmacology and provides insight into the potency/affinity of small molecules.

We presented an approach that can be potentially extended to other human, bacterial or plasmodial targets. The inherent capability of PCM to combine bioactivity data for related targets, even for targets spanning distant phyla, is likely to improve the mining of currently available multi-target bioactivity databases. Similarly, domain-based extrapolation permits in silico target predictions to be extended to non-mammalian orthologous proteins for which less bioactivity data is usually available.

\section{Additional files}

Additional file 1: Training set for the target prediction algorithm.

Additional file 2: InterPro domain annotations for 148 Plasmodium falciparum targets.

Additional file 3: Supplementary Figures and Supplementary Table S3. Additional file 4: Supplementary Figures and Supplementary Table S3.

\section{Competing interests}

The authors declare no competing interests.

\section{Authors' contributions}

SP and ICC designed research. SP and ICC gathered the data, trained the models, and prepared the figures. SP, ICC, APIJ, TM and AB analyzed the results and wrote the paper. All authors read and approved the final manuscript.

\section{Acknowledgements}

ICC thanks the Paris-Pasteur International PhD Programme for funding. ICC and TM thank CNRS and Institut Pasteur for funding. SP and APIJ thank the Netherlands Organisation for Scientific Research (NWO, grant number NWO-017.009-065) and the Prins Bernhard Cultuurfonds for funding. AB thanks Unilever and the European Research Commission (Starting Grant ERC-2013-StG 336159 MIXTURE) for funding.

\section{Author details}

'Department of Chemistry, Centre for Molecular Science Informatics, University of Cambridge, Lensfield Road, CB2 1EW Cambridge, UK. ²Division of Medicinal Chemistry, Leiden Academic Centre for Drug Research, Leiden University, P.O. Box 9502, 2300 RA Leiden, The Netherlands. ${ }^{3}$ Unité de Bioinformatique Structurale, Institut Pasteur and CNRS UMR 3825, Structural Biology and Chemistry Department, 25-28, rue du Dr. Roux, 75724 Paris, France.

Received: 18 November 2014 Accepted: 17 March 2015 Published online: 15 April 2015

\section{References}

1. Jalencas X, Mestres J. On the origins of drug polypharmacology. Med Chem Comm. 2013;4:80. 
2. Lounkine E, Keiser MJ, Whitebread S, Mikhailov D, Hamon J, Jenkins JL, et al. Large-scale prediction and testing of drug activity on side-effect targets. Nature. 2012;486:361-7.

3. Cortes-Ciriano I, Ain QU, Subramanian V, Lenselink EB, Mendez-Lucio O, IJzerman AP, et al. Polypharmacology modelling using proteochemometrics: recent developments and future prospects. Med Chem Comm. 2015;6:24-50 doi: 10.1039/C4MD00216D.

4. Van Westen GJ, Swier RF, Cortes-Ciriano I, Wegner JK, Overington JP, IJzerman AP, et al. Benchmarking of protein descriptor sets in proteochemometric modeling (part 2): modeling performance of 13 amino acid descriptor sets. J Chem inform. 2013;5:42.

5. Poroikov V, Filimonov D, Lagunin A, Gloriozova T, Zakharov A. PASS: identification of probable targets and mechanisms of toxicityt. SAR QSAR Env Res. 2007;18:101-10

6. Nidhi, Glick M, Davies JW, Jenkins JL. Prediction of biological targets for compounds using multiple-category Bayesian models trained on chemogenomics databases. J Chem Inf Model. 2006;46:1124-33.

7. Nigsch F, Bender A, Jenkins JL, Mitchell JBO. Ligand-Target Prediction Using Winnow and Naive Bayesian Algorithms and the Implications of Overall Performance Statistics. J Chem Inf Model. 2008;48:2313-25.

8. Keiser MJ, Roth BL, Armbruster BN, Ernsberger P, Irwin JJ, Shoichet BK. Relating protein pharmacology by ligand chemistry. Nat Biotechnol. 2007;25:197-206.

9. Wale N, Karypis G. Target Fishing for Chemical Compounds using Target-Ligand Activity data and Ranking based Methods. J Chem Inf Model. 2010;49:2190-201.

10. Koutsoukas A, Lowe R, KalantarMotamedi Y, Mussa HY, Klaffke W, Mitchell JBO, et al. In Silico Target Predictions: Defining a Benchmarking Data Set and Comparison of Performance of the Multiclass Naïve Bayes and Parzen-Rosenblatt Window. J Chem Inf Model. 2013;53:1957-66.

11. Van Westen GJP, Wegner JKJ, IJzerman AP, van Vlijmen HWT, Bender A. Proteochemometric modeling as a tool to design selective compounds and for extrapolating to novel targets. Med Chem Comm. 2011;2:16-30.

12. Perlmann $\mathrm{P}$, Troye-Blomberg M. Malaria blood-stage infection and its conyrol by the immune system. Folia Biol (Praha). 2000;46:210-8.

13. Olliaro P. Mode of action and mechanisms of resistance for antimalarial drugs. Pharmacol Ther. 2001;89:207-19.

14. Hecht D, Fogel GB. Modeling the evolution of drug resistance in malaria. J Comput Aided Mol Des. 2012;26:1343-53.

15. Moran M, Guzman J, Ropars A-L. The malaria product pipeline: planning for the future. In: The George Institute for International Health. 2007.

16. ChEMBL - Neglected Tropical Disease. http://www.ebi.ac.uk/chemblntd

17. Gamo F-J, Sanz LM, Vidal J, de Cozar C, Alvarez E, Lavandera J-L, et al. Thousands of chemical starting points for antimalarial lead identification. Nature. 2010;465:305-10

18. Verma R, Tiwari A, Kaur S, Varshney GC, Raghava GPS. Identification of proteins secreted by malaria parasite into erythrocyte using SVM and PSSM profiles. BMC Bio inform. 2008;9:201

19. Jamal S, Periwal V, Scaria V. Predictive modeling of anti-malarial molecules inhibiting apicoplast formation. BMC Bio inform. 2013;14:2105-14.

20. Subramaniam S, Mehrotra M, Gupta D. Support Vector Machine Based Prediction of P. falciparum Proteasome Inhibitors and Development of Focused Library by Molecular Docking. Comb Chem High Throughput Screen. 2011;14:898-907.

21. Vortex D: v2013.03.20719. 2013.

22. Gaulton A, Bellis LJ, Bento AP, Chambers J, Davies M, Hersey A, et al. ChEMBL: a large-scale bioactivity database for drug discovery. Nucleic Acids Res. 2012:40(Database issue):D1100-7

23. Bender A. Databases: Compound bioactivities go public. Nat Chem Biol. 2010;6:309.

24. ChemAxon. Standardizer. 2013.

25. Hunter S, Jones P, Mitchell A, Apweiler R, Attwood TK, Bateman A, et al. InterPro in 2011: new developments in the family and domain prediction database. Nucleic Acid Res. 2012:40(Database issue):D306-12.

26. The Uniprot Consortium. Update on activities at the Universal Protein Resource (UniProt) in 2013. Nucleic Acid Res. 2013:41:D43-7.

27. Bender A, Mussa HY, Glen RC. Molecular Similarity Searching Using Atom Environments, Information-Based Feature Selection, and a Naïve Bayesian Classifier. J Chem Inf Model. 2004;44:170-8.

28. Bender A, Mussa HY, Glen RC. Similarity Searching of Chemical Databases Using Atom Environment Descriptors (MOLPRINT 2D): Evaluation of Performance. J Chem Inf Model. 2004;44:1708-18.

29. Sastry M, Lowrie JF, Dixon SL, Sherman W. Large-scale systematic analysis of $2 \mathrm{D}$ fingerprint methods and parameters to improve virtual screening enrichments. J Chem Inf Model. 2010;50:771-84.
30. O'Boyle NM, Morley C, Hutchison GR. Pybel: a Python wrapper for the OpenBabel cheminformatics toolkit. Chem Cent J. 2008;2:5-11.

31. Crisman TJ, Parker CN, Jenkins JL, Scheiber J, Thoma M, Kang Z, et al. Understanding false positives in reporter gene assays: in silico chemogenimics approaches to prioritize cell-based HTS data. J Chem Inf Model. 2007:47:1319-27.

32. Bender A, Mikhailov D, Glick M, Scheiber J, Davies JW, Cleaver S, et al. Use of Ligand Based Models for Protein Domains To Predict Novel Molecular Targets and Applications To Triage Affinity Chromatography Data. J Proteome Res. 2009;8:2575-85.

33. Prathipati $P, M a ~ N L$, Manjunatha UH, Bender A. Fishing the Target of Antitubercular Compounds: In Silico Target Deconvolution Model Development and Validation. J Proteome Res. 2009;8:2788-98.

34. Murrell DS, Cortes-Ciriano I, van Westen GJP, Stott IP, Malliavin T, Bender A, et al. Chemistry Aware Model Builder (camb): an R Package for Predictive Bioactivity Modeling. 2014. http://github.com/cambDI/camb.

35. Cody V, Galitsky N, Luft JR, Pangborn W, Rosowsky A, Blakley RL. Comparison of two independent crystal structures of human dihydrofolate reductase ternary complexes reduced with nicotinamide adenine dinucleotide phosphate and the very tight-binding inhibitor PT523. Biochemistry. 1997;36:13897-903.

36. Sievers F, Wilm A, Dineen D, Gibson TJ, Karplus K, Li W, et al. Fast, scalable generation of high-quality protein multiple sequence alignments using Clustal Omega. Mol Syst Biol. 2011;7:539.

37. Consonni V, Ballabio D, Todeschini R. Evaluation of model predictive ability by external validation techniques. J Chemometr. 2010;24:194-201.

38. Hawkins DM, Basak SC, Mills D. Assessing Model Fit by Cross-Validation. J Chem Inform Comput Sci. 2003:43:579-86.

39. Tropsha A, Gramatica P, Gombar V. The Importance of Being Earnest: Validation is the Absolute Essential for Successful Application and Interpretation of QSPR Models. QSAR Comb Sci. 2003:22:69-77.

40. Golbraikh A, Tropsha A. Beware of q2! J Mol Graphics Modell. 2002;20:269-76.

41. Brown JB, Okuno Y, Marcou G, Varnek A, Horvath D. Computational chemogenomics: Is it more than inductive transfer? J Comput Aided Mol Des. 2014;28(6):597-618.

42. Ben-Hur A, Ong C. Support vector machines and kernels for computational biology. PLoS Comput Biol. 2008:4:e1000173.

43. Friedman JH. Greedy function approximation: A gradient boosting machine. Ann Stat. 2001;29:1189-232.

44. Rasmussen CE, Williams CKI. Gaussian Processes for Machine Learning, the MIT Press, 2006, ISBN 026218253X. c 2006 Massachusetts Institute of Technology.

45. Breiman L. Random Forests. Mach Learning. 2001;45:5-32.

46. Kuhn M. Building Predictive Models in R Using the caret Package. J Stat Softw. 2008:28:1-26.

47. Spitzmüller A, Mestres J. Prediction of the P. falciparum target space relevant to malaria drug discovery. PLoS Comput Biol. 2013;9:e1003257.

48. Martínez-Jiménez F, Papadatos G, Yang L, Wallace IM, Kumar V, Pieper U, et al. Target prediction for an open access set of compounds active against Mycobacterium tuberculosis. PLoS Comput Biol. 2013;9:e1003253.

49. Clark R, Fox P. Statistical variation in progressive scrambling. J Comput Aided Mol Des. 2004;18:563-76

50. Yuthavong $Y$, Tarnchompoo B, Vilaivan $T$, Chitnumsub $P$, Kamchonwongpaisan S, Charman SA, et al. Malarial dihydrofolate reductase as a paradigm for drug development against a resistance-compromised target. Proc Natl Acad Sci U S A. 2012;109:16823-8.

51. Ersmark K, Samuelsson B, Hallberg A. Plasmepsins as Potential Targets for New Antimalarial Therapy. Med Res Rev. 2006;26:626-66.

52. Marco M, Coterón JM. Falcipain inhibition as a promising antimalarial target. Curr Top Med Chem. 2012;12:408-44.

53. Andrews KT, Tran TN, Wheatley NC, Fairlie DP. Targeting histone deacetylase inhibitors for anti-malarial therapy. Curr Top Med Chem. 2009;9:292-308.

54. Cortes-Ciriano I, Koutsoukas A, Abian O, Glen RC, Velazquez-Campoy A, Bender A. Experimental validation of in silico target predictions on synergistic protein targets. Med Chem Comm. 2013:4:278-88. 